\title{
CLASSIFICATION OF DISCRETE SYSTEMS ON A SQUARE LATTICE
}

\author{
R. Hernández Heredero, D. Levi, and C. Scimiterna
}

We consider the classification up to a Möbius transformation of real linearizable and integrable partial difference equations with dispersion defined on a square lattice by the multiscale reduction around their harmonic solution. We show that the $A_{1}, A_{2}$, and $A_{3}$ linearizability and integrability conditions constrain the number of parameters in the equation, but these conditions are insufficient for a complete characterization of the subclass of multilinear equations on a square lattice.

Keywords: multiscale expansion, difference equation, integrable model, linearizable model

\section{Introduction}

In 1991, Calogero introduced the notion of $S$ - and $C$-integrable equations to characterize those nonlinear partial differential equations (PDEs) that are solvable via an inverse scattering transform and linearizable via a change of variables [1]. Using the multiscale reductive technique, he could show that the nonlinear Schrödinger equation (NLSE)

$$
i \partial_{t} u=K_{2}[u]=\partial_{x x} u+\rho_{2}|u|^{2} u, \quad u=u(x, t),
$$

appears as a universal equation governing the evolution of slowly varying packets of quasimonochromatic waves in weakly nonlinear media featuring dispersion. This evolution is the lowest-order term in the multiscale perturbative expansion of a given PDE. The necessary conditions for the $S$-integrability is that $\rho_{2}$ is real. But if the equation is linearizable, then $\rho_{2}=0$ is the condition for the equation to be linear.

Multiscale techniques have been used to find new $S$-integrable PDEs by considering higher orders in the expansion and to prove that the new nonlinear equations are integrable [2]-[4]. Possibly, the most important example of such a nonlinear PDE is the Degasperis-Procesi equation [5].

As shown in [6]-[8], the introduction of multiple scales on a lattice reduces the given discrete equation either to a local nonlinear partial difference equation by imposing a slow-varying condition or to a PDE when dealing with $\mathbb{C}^{\infty}$ functions. We choose the second alternative here because the integrability and linearizability conditions of the discrete equation, as shown in $[6],[7]$, are preserved only in this case.

It was shown in [6]-[14] that in the case of discrete equations, we have the equivalent of the CalogeroEckhaus theorem [15] stating that a nonlinear dispersive partial difference equation is not $S$-integrable if its multiscale expansion with respect to $\mathbb{C}^{\infty}$ functions does not yield an integrable NLSE at the lowest order. Moreover, it was shown in examples that a multiscale expansion with respect to $\mathbb{C}^{\infty}$ functions of a nonlinear $C$-integrable partial difference equation yields a linear PDE [16]. 


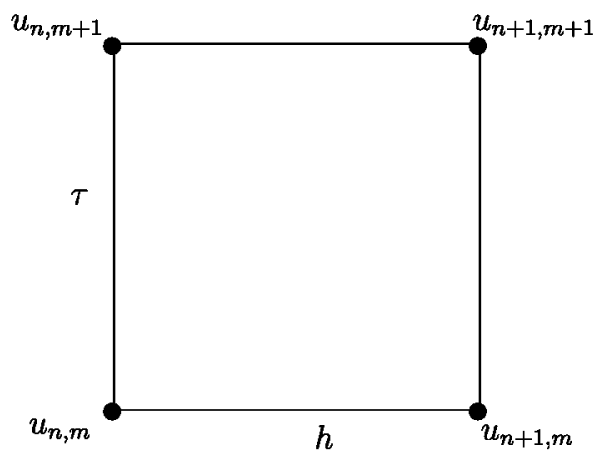

Fig. 1. The $\mathbb{Z}^{2}$ square lattice where the equation $\mathcal{Q}=0$ is defined.

Moreover, in [17], we presented some results on classifying linearizable partial difference equations using the multiscale reduction around their harmonic solution.

Here, we review the results so far obtained on classifying linearizable and integrable partial difference equations on a square lattice around their harmonic solutions. We find necessary conditions for the linearizability and for the integrability of a class of real difference equations in the variable $u: \mathbb{Z}^{2} \rightarrow \mathbb{R}$ and its three nearest-neighbors defined on a $\mathbb{Z}^{2}$ square lattice,

$$
\mathcal{Q}\left(u_{n, m}, u_{n+1, m}, u_{n, m+1}, u_{n+1, m+1} ; \beta_{1}, \beta_{2}, \ldots\right)=0
$$

where the $\beta_{i}$ are real parameters (see Fig. 1). We obtain the linearizability and integrability conditions by a multiscale perturbative expansion, requiring that Eq. (2) be asymptotically equivalent to the NLSE, its hierarchy, and its symmetries or its linearized counterpart.

We assume that Eq. (2) is linear-affine in every variable, and our classification is performed up to the simultaneous Möbius transformations

$$
u_{n, m} \mapsto \frac{A u_{n, m}+B}{C u_{n, m}+D} .
$$

In Sec. 2, we briefly discuss the multiscale expansion for Eq. (2). We show the basic ideas for constructing the integrability conditions ensuring that the given equation is $S$ - or $C$-integrable of the order $A_{j}, j=1,2,3$, i.e., that it asymptotically satisfies the $S$ - or $C$-integrability conditions up to the third, fourth, or fifth order in the perturbation parameter. In Sec. 3, we apply the integrability and linearizability conditions to Eq. (2) and present a sequence of theorems giving the conditions on the constants $\beta_{i}$ under which the system is $A_{1^{-}}, A_{2^{-}}$, or $A_{3}$-integrable or $A_{1^{-}}, A_{2^{-}}$, or $A_{3}$-linearizable. Finally, in Sec. 4 , we present some concluding remarks and discuss open problems.

\section{Expansion of real dispersive partial difference equations}

We now briefly illustrate all the ingredients of the reductive perturbative technique needed for treating difference equations, as presented in $[6],[10]$. We here consider the multiscale expansion in the case of $\mathbb{C}^{\infty}$ functions in order to preserve the linearizability or integrability of the given equation.

Introducing multiple lattice scales under some obvious assumptions on the $\mathbb{C}^{\infty}$ property of the function $u_{n, m}$ and on the radius of convergence of its Taylor expansion for all the $n$ and $m$ shifts involved in difference equation (2), we can write a series representation of the shifted values of $u_{n, m}$ near the point $(n, m)$. Choosing

$$
n_{i}=\varepsilon_{n_{i}} n, \quad \varepsilon_{n_{i}} \doteq N_{i} \varepsilon^{i}, \quad 1 \leq i \leq K_{n}, \quad m_{\sigma}=\varepsilon_{m_{\sigma}} m, \quad \varepsilon_{m_{\sigma}} \doteq M_{\sigma} \varepsilon^{\sigma}, \quad 1 \leq \sigma \leq K_{m},
$$


where the various constants $N_{i}, M_{\sigma}$, and $\varepsilon$ are real numbers, moreover, $K_{n}=1$ and $K_{m}=K$ (eventually, $K=+\infty$ ), we can rewrite the total shift operators $T_{n}$ and $T_{m}$ in terms of partial shift operators $\mathcal{T}_{n}$ and $\mathcal{T}_{m}$ and in terms of differential operators $\mathcal{A}_{m}^{(j)}$ and $\mathcal{A}_{n, m}^{(j)}$ :

$$
\begin{aligned}
& T_{n}=\mathcal{T}_{n} \mathcal{T}_{n_{1}}^{\left(\varepsilon_{n_{1}}\right)}=\mathcal{T}_{n} \sum_{j=0}^{+\infty} \varepsilon^{j} \mathcal{A}_{n}^{(j)}, \quad \mathcal{A}_{n}^{(j)} \doteq \frac{N_{1}^{j}}{j !} \partial_{n_{1}}^{j}, \\
& T_{m}=\mathcal{T}_{m} \prod_{j=1}^{K} \mathcal{T}_{m_{j}}^{\left(\varepsilon_{m_{j}}\right)}=\mathcal{T}_{m} \sum_{j=0}^{+\infty} \varepsilon^{j} \mathcal{A}_{m}^{(j)}, \\
& T_{n} T_{m}=\mathcal{T}_{n} \mathcal{T}_{m} \mathcal{T}_{n_{1}}^{\left(\varepsilon_{n_{1}}\right)} \prod_{j=1}^{K} \mathcal{T}_{m_{j}}^{\left(\varepsilon_{m_{j}}\right)}=\mathcal{T}_{n} \mathcal{T}_{m} \sum_{j=0}^{+\infty} \varepsilon^{j} \mathcal{A}_{n, m}^{(j)} .
\end{aligned}
$$

These operators are given by appropriate combinations of $\left(M_{k}^{j} / j !\right) \partial_{m_{k}}^{j}$ (explicit expressions and formulas can be found in [10]; see [18] for more details). For the function $u_{n, m}=u\left(n, m, n_{1},\left\{m_{\sigma}\right\}_{\sigma=1}^{K}, \varepsilon\right)$, we can assume a double expansion in harmonics and in the perturbation parameter $\varepsilon$,

$$
u_{n, m}=\sum_{j=1}^{+\infty} \sum_{\theta=-j}^{j} \varepsilon^{j} u_{j}^{(\theta)}\left(n_{1}, m_{\sigma}, \sigma \geq 1\right) e^{i \theta(\kappa h n-\omega \tau m)}
$$

with $u_{j}^{(-\theta)}\left(n_{1}, m_{\sigma}, \sigma \geq 1\right)=\bar{u}_{j}^{(\theta)}\left(n_{1}, m_{\sigma}, \sigma \geq 1\right)$, which ensures that $u_{n, m}$ is real and where $h$ and $\tau$ are the lattice spacings in the respective directions $n$ and $m$. With explicit expressions (4) for the shift operators in terms of the derivatives with respect to the slow variables substituted, (2) then becomes an infinite system of coupled PDEs. Moreover, we require that the functions $u_{\gamma}^{(\theta)}$ satisfy the asymptotic conditions $\lim _{n_{1} \rightarrow \pm \infty} u_{j}^{(\theta)}=0$ for all $j$ and $\theta$ and take the index $j \geq 1$ to let any nonlinear part of (2) enter the multiscale expansion as a perturbation.

The multiscale expansion of a nonlinear partial difference equation with respect to $\mathbb{C}^{\infty}$ functions thus yields continuous PDEs. A multiscale integrability or linearizability test therefore requires that the equation be $S$-integrable or $C$-integrable if its multiscale expansion yields the NLSE hierarchy or its linearized version. This becomes obvious when we consider the orders beyond those where the NLSE or its linearized version is obtained for the coefficient of the first harmonic $u_{1}^{(1)}$. Different approaches to the higher-order multiscale expansion of $S$-integrable nonlinear PDEs are contained in [2], [19], [20], and we considered the linearizable case in [11].

We formulate the following definition.

Definition 1. A nonlinear PDE is said to be $S$-integrable if it has a nontrivial Lax pair and consequently infinitely many generalized symmetries.

Following [2], we can then remove all secular terms from the reduced equations order by order, and in agreement with [3], [21], we hence formulate the following theorems.

Theorem 1. A nonlinear dispersive partial difference equation is $C$-integrable only if its multiscale expansion is given by a uniform asymptotic series such that

1. the amplitude $u_{1}^{(1)}$ evolves in the slow times $m_{\sigma}, \sigma \geq 1$, according to the $\sigma$ th equation of the linear Schrödinger hierarchy,

$$
\partial_{m_{\sigma}} u_{1}^{(1)}=(-i)^{(\sigma-1)} B_{\sigma} \partial_{n_{1}}^{\sigma} u_{1}^{(1)}, \quad B_{\sigma} \doteq-\frac{1}{\sigma !} \frac{d^{\sigma} \omega(\kappa)}{d \kappa^{\sigma}},
$$

where $B_{\sigma}$ are constants, and 
2. the amplitudes of the higher perturbations of the first harmonic $u_{j}^{(1)}, j \geq 2$, evolve in the slow times $m_{\sigma}, \sigma \geq 2$, according to certain linear, nonhomogeneous equations with the proper asymptotic boundary conditions taken into account,

$$
\partial_{m_{\sigma}} u_{j}^{(1)}-(-i)^{(\sigma-1)} B_{\sigma} \partial_{n_{1}}^{\sigma} u_{j}^{(1)} \equiv M_{\sigma} u_{j}^{(1)}=f_{\sigma}(j)
$$

for all $j, \sigma \geq 2$, where $B_{\sigma} \partial_{n_{1}}^{\sigma} u_{1}^{(j)}$ is the $\sigma$ th flow of linear Schrödinger hierarchy (6). All other $u_{j}^{(\theta)}$, $\theta \geq 2$, are expressed in terms of differential monomials of $u_{\rho}^{(1)}, \rho \leq j$.

Theorem 2. A nonlinear dispersive partial difference equation is $S$-integrable only if its multiscale expansion is given by a uniform asymptotic series such that

1. the amplitude $u_{1}^{(1)}$ evolves in the slow times $m_{\sigma}, \sigma \geq 1$, according to the $\sigma$ th equation of the nonlinear Schrödinger hierarchy,

$$
\partial_{m_{\sigma}} u_{1}^{(1)}=K_{\sigma}\left[u_{1}^{(1)}\right]
$$

where $K_{\sigma}\left[u_{1}^{(1)}\right]$ is the $\sigma$ th flow of the nonlinear Schrödinger hierarchy, and

2. the amplitudes of the higher perturbations of the first harmonic $u_{j}^{(1)}, j \geq 2$, evolve in the slow times $m_{\sigma}, \sigma \geq 2$, according to certain nonlinear, nonhomogeneous equations with the proper asymptotic boundary conditions taken into account,

$$
M_{\sigma} u_{j}^{(1)}=f_{\sigma}(j), \quad M_{\sigma} \doteq \partial_{t_{\sigma}}-K_{\sigma}^{\prime}\left[u_{1}^{(1)}\right]
$$

for all $j, \sigma \geq 2$. All other $u_{j}^{(\kappa)}, \kappa \geq 2$, are expressed in terms of differential monomials of $u_{\rho}^{(1)}, \rho \leq j$.

In (7) and $(9), f_{\sigma}(j)$ is a nonhomogeneous nonlinear forcing term, the $B_{\sigma}, \sigma \geq 1$, introduced in (6) are complex constants, and $K_{\sigma}^{\prime}[u] v$ is the Frechet derivative of the nonlinear term $K_{\sigma}[u]$ along the direction $v$ defined as $K_{\sigma}^{\prime}[u] v \doteq d K_{\sigma}[u+s v] /\left.d s\right|_{s=0}$, i.e., the linearization near $u$ of $K_{\sigma}[u]$ along the direction $v$. Equations (6) and (8) represent a hierarchy of compatible evolutions for the function $u_{1}^{(1)}$. It is obvious that the operators $M_{\sigma}$ defined in (7) and (9) mutually commute. Therefore, if we fix the index $j \geq 2$ in system of equations (7), (9), then their compatibilities imply the conditions

$$
M_{\sigma} f_{\sigma^{\prime}}(j)=M_{\sigma^{\prime}} f_{\sigma}(j) \quad \forall \sigma, \sigma^{\prime} \geq 2
$$

where because $f_{\sigma}(j)$ and $f_{\sigma^{\prime}}(j)$ are functions of different $u_{j}^{(1)}$ (the perturbations of the coefficients of the fundamental harmonic up to the degree $j-1$ ), the time derivatives $\partial_{t_{\sigma}}$ and $\partial_{t_{\sigma^{\prime}}}$ of those coefficients respectively appearing in $M_{\sigma}$ and $M_{\sigma^{\prime}}$ must be eliminated using evolution equations (6)-(9) up to the index $j-1$. Commutativity conditions (10) turn out to be an integrability test. The explicit forms of these conditions can be found in [17], [22].

\section{Dispersive affine-linear equations on a square lattice}

We consider a real homogeneous affine-linear equation with dispersion defined on a square lattice and belonging to class (2). The most general multilinear equation of class (2) has at most quartic nonlinearity. We introduce the solution $u_{n, m}=K^{n} \Omega^{m}$ in its linear part, where $K=e^{i \kappa}$ and $\Omega=e^{-i \omega(\kappa)}$. We find that 
this equation is dispersive if it is homogeneous and written as

$$
\begin{aligned}
\mathcal{Q}_{ \pm}= & a_{1}\left(u_{n, m} \pm u_{n+1, m+1}\right)+a_{2}\left(u_{n+1, m} \pm u_{n, m+1}\right)+ \\
& +\left(\alpha_{1}-\alpha_{2}\right) u_{n, m} u_{n+1, m}+\left(\alpha_{1}+\alpha_{2}\right) u_{n, m+1} u_{n+1, m+1}+ \\
& +\left(\beta_{1}-\beta_{2}\right) u_{n, m} u_{n, m+1}+\left(\beta_{1}+\beta_{2}\right) u_{n+1, m} u_{n+1, m+1}+ \\
& +\gamma_{1} u_{n, m} u_{n+1, m+1}+\gamma_{2} u_{n+1, m} u_{n, m+1}+ \\
& +\left(\xi_{1}-\xi_{3}\right) u_{n, m} u_{n+1, m} u_{n, m+1}+\left(\xi_{1}+\xi_{3}\right) u_{n, m} u_{n+1, m} u_{n+1, m+1}+ \\
& +\left(\xi_{2}-\xi_{4}\right) u_{n+1, m} u_{n, m+1} u_{n+1, m+1}+\left(\xi_{2}+\xi_{4}\right) u_{n, m} u_{n, m+1} u_{n+1, m+1}+ \\
& +\zeta u_{n, m} u_{n+1, m} u_{n, m+1} u_{n+1, m+1}=0,
\end{aligned}
$$

where $a_{1}, a_{2} \in \mathbb{R} \backslash\{0\},\left|a_{1}\right| \neq\left|a_{2}\right|$, are the coefficients appearing in the linear part and $\alpha_{1}, \alpha_{2}, \beta_{1}, \beta_{2}, \gamma_{1}$, $\gamma_{2}$, and $\xi_{1}, \ldots, \xi_{4}, \zeta$ are eleven real parameters to be determined using the multiscale procedure described in Sec. 2. The linear dispersion relation is given by

$$
\omega(\kappa)=\arctan \left[\frac{\left(a_{1}^{2}-a_{2}^{2}\right) \sin \kappa}{\left(a_{1}^{2}+a_{2}^{2}\right) \cos \kappa+2 a_{1} a_{2}}\right] .
$$

We seek those transformations that leave the class of equations $\mathcal{Q}_{ \pm}$invariant. They give the equivalence conditions for our classification. It is well known that polynomial equations are invariant under simultaneous Möbius transformations $u_{n, m} \mapsto\left(A u_{n, m}+B\right) /\left(C u_{n, m}+D\right)$. But our class of equations is homogeneous with a restriction on the coefficients of the linear part. After a Möbius transformation, a constant term

$$
a_{0}=B^{4} \zeta+2 B^{3} D\left(\xi_{1}+\xi_{2}\right)+B^{2} D^{2}\left[\gamma_{1}+\gamma_{2}+2\left(\alpha_{1}+\beta_{1}\right)\right]+2 B D^{3}\left(a_{1}+a_{2}\right)
$$

appears. Therefore, if we do not want to restrict the coefficients of the equation, then we must set $B=0$ to have $a_{0}=0$. Under the Möbius transformation with $B=0$, the coefficients of $\mathcal{Q}_{ \pm}$become

$$
\begin{aligned}
& a_{1} \mapsto D^{3} a_{1}, \quad a_{2} \mapsto D^{3} a_{2}, \quad \alpha_{1} \mapsto D^{2}\left[\alpha_{1}+C\left(a_{1}+a_{2}\right)\right], \quad \alpha_{2} \mapsto D^{2} \alpha_{2}, \\
& \beta_{1} \mapsto D^{2}\left[\beta_{1}+C\left(a_{1}+a_{2}\right)\right], \quad \beta_{2} \mapsto D^{2} \beta_{2}, \\
& \gamma_{1} \mapsto D^{2}\left(\gamma_{1}+2 C a_{1}\right), \quad \gamma_{2} \mapsto D^{2}\left(\gamma_{2}+2 C a_{2}\right), \\
& \xi_{1} \mapsto D \xi_{1}+\frac{1}{2} C D\left[3 C\left(a_{1}+a_{2}\right)+\gamma_{1}+\gamma_{2}+2\left(\alpha_{1}-\alpha_{2}+\beta_{1}\right)\right], \\
& \xi_{2} \mapsto D \xi_{2}+\frac{1}{2} C D\left[3 C\left(a_{1}+a_{2}\right)+\gamma_{1}+\gamma_{2}+2\left(\alpha_{1}+\alpha_{2}+\beta_{1}\right)\right] \\
& \xi_{3} \mapsto D \xi_{3}+\frac{1}{2} C D\left[C\left(a_{1}-a_{2}\right)+\gamma_{1}-\gamma_{2}+2 \beta_{2}\right], \\
& \xi_{4} \mapsto D \xi_{4}+\frac{1}{2} C D\left[C\left(a_{1}-a_{2}\right)+\gamma_{1}-\gamma_{2}-2 \beta_{2}\right], \\
& \zeta \mapsto \zeta+C^{2}\left[2 C\left(a_{1}+a_{2}\right)+\gamma_{1}+\gamma_{2}+2\left(\alpha_{1}+\beta_{1}\right)\right]+2 C\left(\xi_{1}+\xi_{2}\right) .
\end{aligned}
$$

Hence, our equivalence transformation with respect to which we classify the equation $\mathcal{Q}_{ \pm}$is a restricted Möbius transformation of the form

$$
u_{n, m} \mapsto \frac{u_{n, m}}{C u_{n, m}+D}
$$

We limit ourself here to the case $\mathcal{Q}_{+}$; the case $\mathcal{Q}_{-}$will be considered in a subsequent publication. 
3.1. Linearizable dispersive affine-linear equations on a square lattice in the class $\mathcal{Q}_{+}$. Requiring that the lowest order of the multiscale expansion yield the linear Schrödinger equation, we obtain the following theorem.

Theorem 3. In the lowest order $\varepsilon^{3}$, the necessary conditions for the linearizability of the equations $\mathcal{Q}_{+}$lead to six different classes of equations characterized by different ranges of values of the coefficients of the equation $\mathcal{Q}_{+}$as follows:

- Case 1:

$$
\begin{aligned}
& \alpha_{2}=\beta_{2}=0, \quad \alpha_{1}=\beta_{1}, \quad \gamma_{1}+\gamma_{2}=2 \beta_{1} \\
& \xi_{1}=\xi_{2}=\frac{\left(a_{1}+a_{2}\right)^{2} \gamma_{1} \gamma_{2}+\left(3 a_{1}-2 a_{2}\right) a_{2} \gamma_{1} \beta_{1}-a_{1}\left(2 a_{1}-3 a_{2}\right) \gamma_{2} \beta_{1}}{4 a_{1} a_{2}\left(a_{1}+a_{2}\right)} \\
& \xi_{3}=\xi_{4}=\frac{-\left(a_{1}-a_{2}\right)\left(a_{1}+a_{2}\right)^{2} \gamma_{1} \gamma_{2}-a_{2}\left(-a_{1}^{2}-5 a_{2} a_{1}+2 a_{2}^{2}\right) \gamma_{1} \beta_{1}+a_{1}\left(2 a_{1}^{2}-5 a_{2} a_{1}-a_{2}^{2}\right) \gamma_{2} \beta_{1}}{4 a_{1} a_{2}\left(a_{1}+a_{2}\right)^{2}}
\end{aligned}
$$

- Case 2:

$$
\begin{aligned}
& \alpha_{2}=\beta_{2}=0, \quad \alpha_{1}=\beta_{1}, \quad\left(3 a_{1}-2 a_{2}\right) a_{2}^{2} \gamma_{1}+a_{1}^{2}\left(2 a_{1}-3 a_{2}\right) \gamma_{2}=4 a_{1}\left(a_{1}-a_{2}\right) a_{2} \beta_{1}, \\
& \xi_{1}=\xi_{2}=\frac{\left(a_{1}+a_{2}\right)\left(a_{2}^{2} \gamma_{1}^{2}-a_{1}^{2} \gamma_{2}^{2}\right)+2 a_{2}\left(a_{1}^{2}-2 a_{2}^{2}\right) \gamma_{1} \beta_{1}+2 a_{1}\left(2 a_{1}^{2}-a_{2}^{2}\right) \gamma_{2} \beta_{1}-6 a_{1}\left(a_{1}-a_{2}\right) a_{2} \beta_{1}^{2}}{4 a_{1} a_{2}\left(a_{1}^{2}-a_{2}^{2}\right)}, \\
& \xi_{3}=\xi_{4}=\frac{2 a_{1} a_{2}\left(a_{1}+a_{2}\right)\left(\gamma_{1}-\gamma_{2}\right) \beta_{1}+\left(a_{1}-a_{2}\right)\left(a_{2} \gamma_{1}-a_{1} \gamma_{2}\right)^{2}+2 a_{1} a_{2}\left(a_{2}-a_{1}\right) \beta_{1}^{2}}{4 a_{1} a_{2}\left(a_{1}+a_{2}\right)^{2}},
\end{aligned}
$$

- Case 3:

$$
\begin{aligned}
& \alpha_{2}=\beta_{2}=0, \quad a_{1}=2 a_{2}, \quad \gamma_{1}=\frac{2}{3}\left(\alpha_{1}+\beta_{1}\right), \quad \gamma_{2}=\frac{1}{3}\left(\alpha_{1}+\beta_{1}\right), \\
& \xi_{1}=\xi_{2}=-\frac{-5 \alpha_{1} \beta_{1}+\alpha_{1}^{2}+\beta_{1}^{2}}{6 a_{2}}, \quad \xi_{3}=\xi_{4}=-\frac{\left(\alpha_{1}-2 \beta_{1}\right)\left(2 \alpha_{1}-\beta_{1}\right)}{18 a_{2}},
\end{aligned}
$$

- Case 4:

$$
\begin{aligned}
& \alpha_{2}=\beta_{2}=0, \quad 2 a_{1}=a_{2}, \quad \gamma_{1}=\frac{1}{3}\left(\alpha_{1}+\beta_{1}\right), \quad \gamma_{2}=\frac{2}{3}\left(\alpha_{1}+\beta_{1}\right), \\
& \xi_{1}=\xi_{2}=-\frac{-5 \alpha_{1} \beta_{1}+\alpha_{1}^{2}+\beta_{1}^{2}}{6 a_{1}}, \quad \xi_{3}=\xi_{4}=\frac{\left(\alpha_{1}-2 \beta_{1}\right)\left(2 \alpha_{1}-\beta_{1}\right)}{18 a_{1}},
\end{aligned}
$$

- Case 5:

$$
\begin{aligned}
& \alpha_{2}=\beta_{2}, \quad \alpha_{1}=\beta_{1}, \quad 2 a_{1}=a_{2}, \quad \gamma_{1}=\frac{2 \beta_{1}}{3}, \quad \gamma_{2}=\frac{4 \beta_{1}}{3}, \\
& \xi_{1}=\frac{3 \beta_{1}^{2}-2 \beta_{2} \beta_{1}+\beta_{2}^{2}}{6 a_{1}}, \quad \xi_{2}=\frac{3 \beta_{1}^{2}+2 \beta_{2} \beta_{1}+\beta_{2}^{2}}{6 a_{1}}, \\
& \xi_{3}=-\frac{\beta_{1}^{2}-6 \beta_{2} \beta_{1}+7 \beta_{2}^{2}}{18 a_{1}}, \quad \xi_{4}=-\frac{\beta_{1}^{2}+6 \beta_{2} \beta_{1}+7 \beta_{2}^{2}}{18 a_{1}},
\end{aligned}
$$

- Case 6:

$$
\begin{array}{ll}
\alpha_{2}=-\beta_{2}, \quad \alpha_{1}=\beta_{1}, & a_{1}=2 a_{2}, \quad \gamma_{1}=\frac{4 \beta_{1}}{3}, \\
\xi_{1}=\frac{3 \beta_{1}^{2}+2 \beta_{2} \beta_{1}+\beta_{2}^{2}}{6 a_{2}}, & \xi_{2}=\frac{3 \beta_{1}^{2}-2 \beta_{2} \beta_{1}+\beta_{2}^{2}}{6 a_{2}}, \\
\xi_{3}=\frac{\beta_{1}^{2}+6 \beta_{2} \beta_{1}+7 \beta_{2}^{2}}{18 a_{2}}, & \xi_{4}=\frac{\beta_{1}^{2}-6 \beta_{2} \beta_{1}+7 \beta_{2}^{2}}{18 a_{2}} .
\end{array}
$$


The six corresponding subclasses of equations are invariant under restricted Möbius transformations (22).

Cases 1-6 are also $A_{2} C$-integrable because there is no linearizability condition in the order $\varepsilon^{4}$.

As a consequence of Theorem 3, we can state the following obvious, but important, corollary.

Corollary 1. If the coefficients $a_{1}, a_{2}, \alpha_{1}, \alpha_{2}, \beta_{1}, \beta_{2}, \gamma_{1}, \gamma_{2}$, and $\xi_{1}, \ldots, \xi_{4}$ of the equations $\mathcal{Q}_{+}$do not satisfy one of conditions (23)-(28), then $\mathcal{Q}_{+}$is not linearizable.

We note that the trivial linearizability condition $\alpha_{1}=\alpha_{2}=\beta_{1}=\beta_{2}=\gamma_{1}=\gamma_{2}=\xi_{1}=\xi_{2}=\xi_{3}=\xi_{4}=0$ is contained in (23)-(28).

It is particularly interesting to consider the case where the equation has at most quadratic nonlinearity. In this case, we obtain the following theorem.

Theorem 4. If $\xi_{1}=\xi_{2}=\xi_{3}=\xi_{4}=0$ in the equations $\mathcal{Q}_{+}$, then the lowest linearizability conditions are

- Cases 1a and 1b:

$$
\begin{aligned}
& \alpha_{2}=\beta_{2}=0, \quad \alpha_{1}=\beta_{1}, \quad a_{1}=(1 / 4)(7 \pm \sqrt{33}) a_{2}, \\
& \gamma_{1}=(1 \mp \sqrt{3 / 11}) \beta_{1}, \quad \gamma_{2}=(1 \pm \sqrt{3 / 11}) \beta_{1},
\end{aligned}
$$

- Cases 2a and 2b:

$$
\begin{aligned}
& \alpha_{2}=\beta_{2}=0, \quad \alpha_{1}=\beta_{1}, \quad a_{1}=(3 / 4+1 / \sqrt{2} \pm(1 / 2) \sqrt{1 / 4+3 \sqrt{2}}) a_{2} \\
& \gamma_{1}=\mp 2 \sqrt{(1 / 41)(19+18 \sqrt{2})} \beta_{1}, \quad \gamma_{2}= \pm 2 \sqrt{(1 / 41)(19+18 \sqrt{2})} \beta_{1} .
\end{aligned}
$$

Cases $1 \mathrm{a}$ and $1 \mathrm{~b}$ are contained in case 1 , and cases $2 \mathrm{a}$ and $2 \mathrm{~b}$ are contained in case 2 .

We now consider the $\varepsilon^{5}$ terms of the expansion for cases $1-6$. We have the following theorem.

Theorem 5. The most general $A_{3} C$-integrable equation is represented as

$$
\begin{aligned}
& \alpha_{1}=\beta_{1}=\frac{\left(a_{1}+a_{2}\right) \gamma_{1}}{2 a_{1}}, \quad \alpha_{2}=\beta_{2}=0, \quad \gamma_{2}=\frac{a_{2} \gamma_{1}}{a_{1}}, \\
& \xi_{1}=\xi_{2}=\frac{3\left(a_{1}+a_{2}\right) \gamma_{1}^{2}}{8 a_{1}^{2}}, \quad \xi_{3}=\xi_{4}=\frac{\left(a_{1}-a_{2}\right) \gamma_{1}^{2}}{8 a_{1}^{2}} .
\end{aligned}
$$

Case (29) is the intersection of cases (23) and (24). As a consequence of result (29), we find that an equation of the form $\mathcal{Q}_{+}$satisfying Theorem 4, i.e., dispersive with at most quadratic nonlinearity, is never $C$-integrable. By a restricted Möbius transformation

$$
u_{n, m}=\frac{\alpha v_{n, m}+\beta}{\gamma v_{n, m}+\delta}, \quad \alpha=2 a_{1} \delta, \quad \beta=0, \quad \gamma=-\gamma_{1} \delta
$$

we can obtain the canonical form of equations belonging to case (29):

$$
v_{n, m}+v_{n+1, m+1}+\epsilon\left(v_{n+1, m}+v_{n, m+1}\right)+\zeta^{\prime} v_{n, m} v_{n+1, m} v_{n, m+1} v_{n+1, m+1}=0,
$$

where $\epsilon \doteq a_{2} / a_{1} \neq 0, \pm 1$ and $\zeta^{\prime} \doteq-2\left[\left(a_{1}+a_{2}\right) \gamma_{1}^{3}-4 a_{1}^{3} \zeta\right] / a_{1}$.

The following theorem is easily proved. 
Theorem 6. Equations $\mathcal{Q}_{+}$satisfying $A_{3} C$-integrability (29) can be linearized by a real Möbius transformation if and only if $\zeta=\left(a_{1}+a_{2}\right) \gamma_{1}^{3} / 4 a_{1}^{3}$ corresponding to $\zeta^{\prime}=0$ in (31). The linearizing transformation is given in (30), and the resulting linearized equation is

$$
v_{n, m}+v_{n+1, m+1}+\epsilon\left(v_{n+1, m}+v_{n, m+1}\right)=0 .
$$

Equation (32) is the most general linear dispersive equation defined on the square.

3.2. Integrable dispersive affine-linear equations on the square lattice in the class $\mathcal{Q}_{+}$. In the lowest order $\varepsilon^{3}$ of the integrability conditions, we obtain the following theorem.

Theorem 7. Necessary conditions in the lowest order for the integrability of the equations $\mathcal{Q}_{+}$are

- Case $1(\mathcal{N}=8)$ :

$$
\alpha_{2}=\beta_{2}=0, \quad \xi_{1}=\xi_{2}, \quad \xi_{3}=\xi_{4},
$$

- Case $2(\mathcal{N}=6)$ :

$$
\begin{aligned}
& \alpha_{2}=\beta_{2}, \quad \alpha_{1}=\beta_{1}, \quad a_{1}=2 a_{2}, \\
& \gamma_{1}=2 \gamma_{2}, \quad a_{1}\left(\xi_{1}-\xi_{2}\right)=-a_{1}\left(\xi_{3}-\xi_{4}\right)=-2 \alpha_{2} \gamma_{2},
\end{aligned}
$$

- Case $3(\mathcal{N}=6)$ :

$$
\begin{aligned}
& \alpha_{2}=-\beta_{2}, \quad \alpha_{1}=\beta_{1}, \quad a_{2}=2 a_{1}, \\
& \gamma_{2}=2 \gamma_{1}, \quad a_{1}\left(\xi_{1}-\xi_{2}\right)=a_{1}\left(\xi_{3}-\xi_{4}\right)=-\alpha_{2} \gamma_{1},
\end{aligned}
$$

- Case $4(\mathcal{N}=7)$ :

$$
\begin{aligned}
& a_{2} \alpha_{1}=a_{2} \beta_{1}=\frac{1}{2}\left(a_{1}+a_{2}\right) \gamma_{2}, \quad a_{2} \gamma_{1}=a_{1} \gamma_{2}, \\
& a_{1}\left(\xi_{1}-\xi_{2}\right)=-\alpha_{2} \gamma_{1}, \quad a_{1}\left(\xi_{3}-\xi_{4}\right)=\beta_{2} \gamma_{1},
\end{aligned}
$$

- Case $5(\mathcal{N}=7)$ :

$$
\begin{aligned}
& \left(a_{2}-a_{1}\right) \beta_{2}=\left(a_{2}+a_{1}\right) \alpha_{2}, \quad 2 a_{1} a_{2}\left(a_{1}-a_{2}\right) \alpha_{1}=\left(a_{1}+a_{2}\right)\left(\gamma_{2} a_{1}^{2}-\gamma_{1} a_{2}^{2}\right), \\
& 2 a_{1} a_{2} \beta_{1}=\gamma_{1} a_{2}^{2}+\gamma_{2} a_{1}^{2}, \quad\left(a_{2}-a_{1}\right)\left(\xi_{1}-\xi_{2}\right)=\left(\gamma_{1}-\gamma_{2}\right) \alpha_{2}, \\
& \left(a_{2}-a_{1}\right)^{2}\left(\xi_{3}-\xi_{4}\right)=\left[\gamma_{2}\left(a_{2}-3 a_{1}\right)-\gamma_{1}\left(a_{1}-3 a_{2}\right)\right] \alpha_{2},
\end{aligned}
$$

- Case $6(\mathcal{N}=7)$ :

$$
\begin{aligned}
& \left(a_{2}+a_{1}\right) \beta_{2}=\left(a_{2}-a_{1}\right) \alpha_{2}, \quad 2 a_{1} a_{2} \alpha_{1}=\gamma_{1} a_{2}^{2}+\gamma_{2} a_{1}^{2}, \\
& 2 a_{1} a_{2}\left(a_{1}-a_{2}\right) \beta_{1}=\left(a_{1}+a_{2}\right)\left(\gamma_{2} a_{1}^{2}-\gamma_{1} a_{2}^{2}\right), \\
& \left(a_{2}^{2}-a_{1}^{2}\right)\left(\xi_{1}-\xi_{2}\right)=\left[\gamma_{1}\left(a_{1}-3 a_{2}\right)-\gamma_{2}\left(a_{2}-3 a_{1}\right)\right] \alpha_{2}, \\
& \left(a_{1}+a_{2}\right)\left(\xi_{3}-\xi_{4}\right)=\left(\gamma_{2}-\gamma_{1}\right) \alpha_{2} .
\end{aligned}
$$

The number $\mathcal{N}$ denotes the number of free parameters contained in the resulting nonlinear lattice equation. The six corresponding subclasses of equations are invariant under restricted Möbius transformations (22).

As in the case of linearizable equations, we can formulate the following corollary. 
Corollary 2. If the coefficients $a_{1}, a_{2}, \alpha_{1}, \alpha_{2}, \beta_{1}, \beta_{2}, \gamma_{1}, \gamma_{2}$, and $\xi_{1}, \ldots, \xi_{4}$ of the equation $\mathcal{Q}_{+}$do not satisfy one of conditions (33)-(35), then $\mathcal{Q}_{+}$is not integrable.

If $\xi_{1}=\xi_{2}=\xi_{3}=\xi_{4}=0$ in an equation $\mathcal{Q}_{+}$, then the lowest-order necessary conditions for the resulting equation to be integrable are

- Case 1: $\alpha_{2}=\beta_{2}=0$,

- Case 2: $\alpha_{2}=\beta_{2}, \alpha_{1}=\beta_{1}, a_{1}=2 a_{2}, \gamma_{1}=\gamma_{2}=0$,

- Case 3: $\alpha_{2}=-\beta_{2}, \alpha_{1}=\beta_{1}, 2 a_{1}=a_{2}, \gamma_{1}=\gamma_{2}=0$,

- Case 4: $\alpha_{1}=\beta_{1}, \gamma_{1}=\gamma_{2}=0$.

These cases are just the corresponding subsets of cases 1-4 in Theorem 7 composed of quadratic equations. Cases 5 and 6 in Theorem 7 do not produce quadratic subcases.

We turn to the order $\varepsilon^{4}$ in the perturbation expansion. We then obtain the following $A_{2}$ integrability classification result.

Theorem 8. The necessary and sufficient conditions for the $\varepsilon^{4}$-asymptotic S-integrability are

- Case 1:

$$
\alpha_{2}=\beta_{2}=0, \quad \xi_{1}=\xi_{2}, \quad \xi_{3}=\xi_{4},
$$

- Case 4:

$$
\begin{aligned}
& \alpha_{1}=\beta_{1}=\frac{\left(a_{1}+a_{2}\right) \gamma_{1}}{2 a_{1}}, \quad \gamma_{2}=\frac{a_{2} \gamma_{1}}{a_{1}}, \\
& a_{1}\left(\xi_{1}-\xi_{2}\right)=-\alpha_{2} \gamma_{1}, \quad a_{1}\left(\xi_{3}-\xi_{4}\right)=\beta_{2} \gamma_{1} .
\end{aligned}
$$

Hence, in the order $\varepsilon^{4}$, cases 1 and 4 in Theorem 7 pass the test, while cases $2,3,5$, and 6 become subcases of 1 and 4 .

In the order $\varepsilon^{5}$ of the perturbation expansion, we have the following $A_{3}$-integrability classification result.

Theorem 9. Up to restricted Möbius transformation (22), the only $\varepsilon^{5}$-asymptotic $S$-integrable cases are given by the normalized equations

$$
\begin{aligned}
v_{n, m} & +v_{n+1, m+1}+2\left(v_{n+1, m}+v_{n, m+1}\right)+v_{n+1, m} v_{n, m+1}\left(\tau_{1}+\tau_{2}\right)+ \\
& +\left(v_{n+1, m} v_{n+1, m+1}+v_{n, m} v_{n, m+1}\right) \tau_{2}+\left(v_{n, m+1} v_{n+1, m+1}+v_{n, m} v_{n+1, m}\right) \tau_{1}+ \\
& +v_{n+1, m} v_{n, m+1}\left(v_{n, m}+v_{n+1, m+1}\right) \tau_{1} \tau_{2}=0, \\
v_{n, m} & +v_{n+1, m+1}+\frac{1}{2}\left(v_{n+1, m}+v_{n, m+1}\right)+v_{n+1, m} v_{n, m+1}\left(\tau_{1}+\tau_{2}\right)+ \\
& +\left(v_{n+1, m} v_{n+1, m+1}+v_{n, m} v_{n, m+1}\right) \tau_{2}+\left(v_{n, m+1} v_{n+1, m+1}+v_{n, m} v_{n+1, m}\right) \tau_{1}+ \\
& +2 v_{n, m} v_{n+1, m+1}\left(v_{n+1, m}+v_{n, m+1}\right) \tau_{1} \tau_{2}+ \\
& +4 v_{n, m} v_{n+1, m} v_{n, m+1} v_{n+1, m+1}\left(\tau_{1}+\tau_{2}\right) \tau_{1} \tau_{2}=0,
\end{aligned}
$$




$$
\begin{aligned}
v_{n, m} & +v_{n+1, m+1}+\epsilon\left(v_{n+1, m}+v_{n, m+1}\right)+ \\
& +s\left[\epsilon v_{n+1, m} v_{n, m+1}\left(v_{n, m}+v_{n+1, m+1}\right)+v_{n, m} v_{n+1, m+1}\left(v_{n+1, m}+v_{n, m+1}\right)\right]+ \\
& +\zeta^{\prime} v_{n, m} v_{n+1, m} v_{n, m+1} v_{n+1, m+1}=0 \\
v_{n, m} & +v_{n+1, m+1}+\epsilon\left(v_{n+1, m}+v_{n, m+1}\right)+ \\
& +s\left[v_{n+1, m} v_{n, m+1}\left(v_{n, m}+v_{n+1, m+1}\right)+\epsilon v_{n, m} v_{n+1, m+1}\left(v_{n+1, m}+v_{n, m+1}\right)\right]+ \\
& +\zeta^{\prime} v_{n, m} v_{n+1, m} v_{n, m+1} v_{n+1, m+1}=0, \\
v_{n, m} & +v_{n+1, m+1}+\epsilon\left(v_{n+1, m}+v_{n, m+1}\right)+v_{n, m} v_{n+1, m+1}-v_{n+1, m} v_{n, m+1}+ \\
& +\left(1-\frac{1}{\epsilon}\right)\left[v_{n+1, m} v_{n, m+1}\left(v_{n, m}+v_{n+1, m+1}\right)-v_{n, m} v_{n+1, m+1}\left(v_{n+1, m}+v_{n, m+1}\right)\right]+ \\
& +\left(1-\frac{1}{\epsilon^{2}}\right) v_{n, m} v_{n+1, m} v_{n, m+1} v_{n+1, m+1}=0 .
\end{aligned}
$$

In (39) and (40), $\tau_{1}=0,1$; if $\tau_{1}=0$, then $\tau_{2}=1$. In (41) and (42), $s=0, \pm 1$ and $\epsilon \neq 0, \pm 1$. In (43), $\epsilon \neq 0, \pm 1,2,1 / 2$.

\section{Conclusions}

We have completely classified linearizable and integrable real dispersive partial difference equations belonging to the $\mathcal{Q}_{+}$class using multiscale expansions around a periodic discrete wave solution of the linearized equation up to the fifth order in the perturbation expansion parameter $\varepsilon$. These calculations show that the multiscale expansion can be effectively used to classify asymptotically linearizable and integrable discrete equations.

The obtained $\varepsilon^{5}$-linearizable system depends on four parameters, only two of which are essential, as can be seen from (31). Requiring that the obtained equation be explicitly linearized by a Möbius transformation, we reduce the parameters to a single parameter. Proving that the equation $\mathcal{Q}_{+}$satisfying just $A_{3} C$-integrability conditions (29) is linearizable is still an open problem. Perhaps, by passing to higher orders in the perturbation expansion, we could fix the parameter $\zeta$ according to Theorem 6 . Some further results indicating that (29) or (31) do not provide linearizable equations can be found in [23].

In the asymptotically $S$-integrable case, the $A_{3}$-integrability conditions provide five Möbius-invariant dispersive partial difference equations belonging to the $\mathcal{Q}_{+}$class depending on a reduced number of free parameters, at most two. Equations (41) and (42) with $s=0$ reduce to (31); moreover, with $\zeta^{\prime}=0$, they were studied in [24], [25] and shown to be $S$-integrable. The other cases, so far as we know, are new, and their integrability must be proved by other techniques (because unless we can go to an infinite order, the multiscale expansion technique provides only necessary, not sufficient, conditions).

We are also studying the $\mathcal{Q}_{-}$case. In the lowest order in the perturbative parameter in this case, we obtain a nonlinear system of PDEs relating the fundamental harmonic to the zeroth harmonic. The solution of this equation is the key ingredient in the classification of this class of equations, which contains all dispersive equations belonging to the ABS classification of multilinear equations on the square [26].

A more basic interesting class of partial difference equations that could be considered is that of the equations defined on a triangle, i.e., equations in the variable $u: \mathbb{Z}^{2} \rightarrow \mathbb{R}$ and its two nearest neighbors,

$$
\mathcal{Q}\left(u_{n, m}, u_{n+1, m}, u_{n, m+1} ; \beta_{1}, \beta_{2}, \ldots\right)=0
$$


where the $\beta_{i}$ are real parameters. It is easy to see that no harmonic solution of the linear part of (44) is available for them. The multiscale expansion presented here is therefore not available in this case, and other techniques should be used. The integrable equations could be classified using the formal symmetry approach (see [24] for the case on the square), while the linearizable equations could be obtained by requiring that a linearizing transformation exist (see [23] for the case on the square). Work on this is in progress.

Acknowledgments. One of the authors (D. L.) thanks R. Hernández Heredero for his support during the author's stay in Madrid. One of the authors (R. H. H.) thanks D. Levi and the INFN, Sezione Roma Tre, for their support during the author's visits to Rome.

This work is supported in part by the Italian Ministry of Education and Research and PRIN "Continuous and discrete nonlinear integrable evolutions: From water waves to symplectic maps" from 2010.

\section{REFERENCES}

1. F. Calogero, "Why are certain nonlinear PDEs both widely applicable and integrable?" in: What is Integrability? (V. E. Zakharov, ed.), Springer, Berlin (1991).

2. A. Degasperis, S. V. Manakov, and P. M. Santini, Phys. D, 100, 187-211 (1997).

3. A. Degasperis and M. Procesi, "Asymptotic integrability," in: Symmetry and Perturbation Theory, SPT98 (A. Degasperis and G. Gaeta, eds.), World Scientific, Singapore (1999), pp. 23-37; A. Degasperis, "Multiscale expansion and integrability of dispersive wave equations," in: Integrability (Lect. Notes Phys., Vol. 767, A. V. Mikhailov, ed.), Springer, Berlin (2009), pp. 215-244.

4. Y. Kodama and A. V. Mikhailov, "Obstacles to asymptotic integrability," in: Algebraic Aspects of Integrable Systems (Prog. Nonlinear Diff. Eq. Their Appl., Vol. 26, A. S. Fokas and I. M. Gelfand, eds.), Birkhäuser, Boston, Mass. (1996), pp. 173-204; Y. Hiraoka and Y. Kodama, "Normal form and solitons," in: Integrability (Lect. Notes Phys., Vol. 767, A. V. Mikhailov, ed.), Springer, Berlin (2009), pp. 175-214.

5. A. Degasperis, D. D. Holm, and A. Hone, Theor. Math. Phys., 133, 1463-1474 (2002).

6. R. Hernández Heredero, D. Levi, M. Petrera, and C. Scimiterna, J. Phys. A, 41, 315208 (2008).

7. R. Hernández Heredero, D. Levi, M. Petrera, and C. Scimiterna, J. Nonlinear Math. Phys., 15 (Suppl. 3), 323-333 (2008).

8. D. Levi, J. Phys. A, 38, 7677-7689 (2005); arXiv:nlin/0505061v1 (2005).

9. M. Agrotis, S. Lafortune, and P. G. Kevrekidis, Discrete Contin. Dyn. Syst., Suppl., 22-29 (2005).

10. R. Hernández Heredero, D. Levi, M. Petrera, and C. Scimiterna, J. Phys. A, 40, F831-F840 (2007).

11. J. Leon and M. Manna, J. Phys. A, 32, 2845-2869 (1999).

12. D. Levi and R. Hernández Heredero, J. Nonlinear Math. Phys., 12 (Suppl. 1), 440-448 (2005).

13. D. Levi and M. Petrera, J. Math. Phys., 47, 043509 (2006); arXiv:math-ph/0510084v1 (2005).

14. S. W. Schoombie, J. Comput. Phys., 101, 55-70 (1992).

15. F. Calogero and W. Eckhaus, Inverse Problems, 3, L27-L32, 229-262 (1987); 4, 11-33 (1987); F. Calogero, A. Degasperis, and X. Ji, J. Math. Phys., 42, 2635-2652 (2001); 41, 6399-6443 (2000); F. Calogero and A. Maccari, "Equations of nonlinear Schrödinger type in $1+1$ and $2+1$ dimensions obtained from integrable PDEs," in: Inverse Problems: An Interdisciplinary Study (Adv. Electronics Electron Phys., Vol. 19, P. C. Sabatier, ed.), Acad. Press, London (1987), pp. 463-480.

16. C. Scimiterna and D. Levi, SIGMA, 1006, 070 (2010); arXiv:1005.5288v2 [nlin.SI] (2010).

17. R. Hernández Heredero, D. Levi, and C. Scimiterna, J. Phys. A, 43, 502002 (2010); arXiv:1011.0141v1 [nlin.SI] (2010).

18. C. Scimiterna, "Multiscale techniques for nonlinear difference equations," Doctoral dissertation, http://www. fis.uniroma3.it/dottorato_tesi/Scimiterna_SCIMITERNA.pdf, Roma Tre Universitá Degli Studi, Rome (2009).

19. Y. Kodama, Phys. Lett. A, 107, 245-249 (1985).

20. Y. Kodama, "Nearly integrable systems: Normal form and solitons," in: Nonlinear Evolutions (Proc. 4th Workshop on Nonlinear Evolution Equations and Dynamical Systems, Balaruc-les-Bains, 11-25 June 1987, J. J. P. Leon, ed.), World Scientific, Teaneck, N. J. (1988), pp. 559-570. 
21. M. Procesi, "Non linear waves, multiscale methods, and integrability master degree," Doctoral dissertation, Physics Department, "La Sapienza" University, Rome (1997).

22. D. Levi and C. Scimiterna, Appl. An., 89, 507-527 (2010).

23. D. Levi and C. Scimiterna, SIGMA, 1107, 079 (2011).

24. D. Levi and R. I. Yamilov, J. Phys. A, 42, 454012 (2009); arXiv:0902.4421v1 [nlin.SI] (2009); 44, 145207 (2011); arXiv:1011.0070v2 [nlin.SI] (2010).

25. C. Scimiterna, B. Grammaticos, and A. Ramani, J. Phys. A, 44, 032002 (2011).

26. V. E. Adler, A. I. Bobenko, and Yu. B. Suris, Commun. Math. Phys., 233, 513-543 (2003); arXiv:nlin/0202024v2 (2002). 